\title{
LA RESPONSABILIDAD SOCIAL DE LA INDUSTRIA DE LOS VIDEOJUEGOS: UNA APROXIMACIÓN DESDE LOS CONTENIDOS
}

\section{The Social Responsibility of the Video Games Industry: An approach from the contents}

\author{
Florina Guadalupe Arredondo Trapero ${ }^{1}$ y José Carlos Vázquez Parra ${ }^{2}$
}

Recibido: 22/8/2017 • Aprobado: 24/9/2017

DOI: http://dx.doi.org/10.22206/cys.2017.v42i4.pp31-39

\begin{abstract}
Resumen
La industria del entretenimiento es una de las áreas a las que mayor atención se debe prestar en cuanto a la evaluación de sus contenidos, ya que, en su mayoría, los consumidores son jóvenes con una madurez relativa. Aunque se tienen las herramientas para evitar el consumo de material inapropiado, estas resultan poco útiles debido al desconocimiento o a la poca importancia que ponen los padres de familia en la revisión de este tipo de productos. Por ende, gran parte de la responsabilidad tiende a recaer en la industria que produce y oferta estos juegos, por lo que el manejo de contenidos que no se ajusta a los fines mismos de los videojuegos puede considerarse como acción irresponsable hacia sus grupos de interés, no respetando sus legítimas demandas. El presente ensayo tiene el objetivo de reflexionar sobre las implicaciones del contenido de los videojuegos, tomando en cuenta el impacto que tiene este tipo de productos en sus usuarios $y$, por consiguiente, la responsabilidad social que implica para las empresas dedicadas a esta industria.
\end{abstract}

Palabras clave: responsabilidad; videojuegos; educación; violencia; industria de entretenimiento.

1. Profesora de la Escuela de Humanidades y Educación del Tecnológico de Monterrey en Monterrey. México

ORCID: 0000-0003-4909-0329

E-mail: farredon@itesm.mx

2. Profesor de la Escuela de Humanidades y Educación del Tecnológico de Monterrey en Guadalajara

ORCID: 0000-0001-9197-7826

E-mail: jcvazquezp@itesm.mx

\begin{abstract}
The entertainment industry is one of the areas in which more attention has to be paid to the evaluation of its contents, since, for the most part, consumers are young people with relative maturity. Although they have tools to avoid the consumption of inappropriate material, these are little useful, because of the ignorance or little importance that parents put in reviewing this type of products. Therefore, much of the responsibility tends to fall on the industry that produces and offers these games, considering that the handling of content that does not fit the very purpose of video games can be considered as irresponsible actions towards its stakeholders, not respecting their legitimate demands. Therefore, this essay aims to reflect on the implications of the content of video games, taking into account the impact that these types of products have on their users and the fact that they imply a social responsibility for companies dedicated to this industry.
\end{abstract}

Keywords: responsibility; videogames; education; violence; entertainment industry. 


\section{Introducción}

En sus inicios, los videojuegos tenían un formato muy básico, las historias eran simples y los gráficos eran bidimensionales. El rápido avance en tecnología computacional ha permitido a los diseñadores de los videojuegos producir cada vez mejores efectos gráficos, que hacen a la realidad virtual más cercana a la realidad real (Simone $\&$ López, 2008). Lo anterior ha llevado incluso a discutir la posibilidad de ver a la realidad virtual como lo real, y a la realidad real como una pseudo-realidad de la que el ser humano escapa para vivir en el ámbito de lo virtual.

Este cuestionamiento no es algo nuevo, ya que fueron preguntas igualmente planteadas por Platón (2007), en su Alegoría de la caverna. Para este filósofo, lo real es el mundo de las ideas, puesto que nuestros sentidos nos engañan y somos incapaces de percibir la realidad a través de ellos. En el ámbito de los videojuegos esta situación ha sido también analizada y criticada:

...El gamer puede perder la dimensión de la realidad real y preferir estar en ese mundo virtual, en donde se siente más cómodo, su avatar ahí nació y creció. El efecto es tal que el gamer puede llegar a olvidarse de tener una vida social común e interactuar con las personas físicamente, crea una burbuja en la cual se encierra y evade la realidad que le rodea (García, 2009, p. 9).

Por otra parte, los videojuegos en línea permiten la posibilidad de crear no solo una realidad virtual, sino incluso una sociedad virtual dentro del propio videojuego. De acuerdo con Cira (2009): "gracias a la posibilidad de interactuar con otros usuarios mediante la experiencia de videojuegos en línea es posible conocer a más personas que comparten la misma afición y en ocasiones también los mismos gustos por ciertas cosas, como música, comida, artes y demás" (p. 12).
Tal como puede analizarse con los avances tecnológicos logrados, hay una realidad virtual que deja de ser "idea", basada en el idealismo platónico (Platón, 2007), para pasar a ser una realidad que se percibe por los sentidos, vinculada a un realismo aristotélico (Aristóteles, 1999), logrando una aproximación o similitud a una realidad que igualmente también es percibida por los sentidos. En otras palabras, las fronteras entre el mundo real y el virtual tienden a desaparecer también desde el punto de vista filosófico. Por ende, ¿tendrá la vida virtual de los jugadores un impacto en su vida real?, ¿̧será necesario que la industria de los videojuegos considere el posible impacto de sus productos como parte de su responsabilidad social?

Este artículo tiene el objetivo de presentar las implicaciones del contenido de los videojuegos, tomando en cuenta la influencia que tiene este tipo de productos en sus usuarios $y$, en consecuencia, la responsabilidad social que implica para las empresas dedicadas a esta industria. La justificación del porqué reflexionar sobre estos puntos surge a partir de que los videojuegos conforman una de las industrias creativas con mayor auge y crecimiento en los últimos años, ubicándose como la segunda área de mayor importancia, solo superada por la industria del cine (McCormick, 2001). La proliferación de juegos nuevos, productos adicionales y su creciente demanda hace necesario abrir la reflexión acerca de las implicaciones que esto genera, resultando pertinente considerar las exigencias sociales que el propio proceso demanda a sus diseñadores.

\section{Marco teórico \\ Videojuegos: una industria creciente}

No se puede negar que la industria de los videojuegos está relacionada inevitablemente con el desarrollo de la tecnología para uso personal. El equipamiento en los hogares tanto de computadoras personales, redes, y acceso a equipos, así como 
el bajo costo de la tecnología, contribuyen a que niños, jóvenes y adultos tengan un importante poder de consumo de este tipo de productos (Simone \& López, 2008). Los niños que fueron parte de la generación de videojuegos en los años noventa han conservado su predilección por seguir jugando en su tiempo de ocio, ampliando la población de consumo y sumándose a las nuevas generaciones que cuentan con un interés cada vez más sofisticado en lo que la industria puede ofrecerles (Peláez, 2009). Se estima que el $66 \%$ de los jugadores frecuentes tiene más de 18 años de edad (Jenkins, 2010).

La industria de los videojuegos es la rama de la economía que se encarga del desarrollo, la distribución, la mercadotecnia, la venta y el hardware de los videojuegos. Desde hace algunos años ha estado creciendo exponencialmente y junto con los avances tecnológicos se volvió una de las fuentes de entretenimiento más buscadas en la actualidad (Rodríguez \& Pestano, 2012). Nació en 1940 junto con las primeras supercomputadoras, después de la Segunda Guerra Mundial, y fue tal su impacto que rápidamente creó un cambio en la forma en que se divertirían las siguientes generaciones (Peláez, 2009).

En general, la industria ha tenido un crecimiento constante y un avance impresionante; aprovechando cada gota de nuevas tecnologías para proveer de productos cada vez más novedosos y realistas (Garfías, 2010). Como todas las demás industrias, la industria de los videojuegos no ha pasado desapercibida en cuanto a los problemas y las críticas, enfocándose la mayoría de los señalamientos en el contenido al que exponen a sus jugadores, que en algunas ocasiones ha llegado a incluir temáticas de violencia, sexo y consumo de sustancias (Sinde, Medrano \& Martínez, 2015).

Esta situación es la que llega a cuestionar los fines últimos de la industria de los videojuegos o incluso de sus diseñadores y creadores de contenido, pues si el fin último de un juego es divertir a sus usuarios, que en su mayoría son jóvenes, ¿qué necesidad hay de incluir situaciones que desafíen sus valores o su percepción de la realidad?

\section{Contenidos con fines confusos}

De acuerdo con Takahashi (2004), para algunos diseñadores la ética o la falta de ella no está vinculada propiamente al videojuego, sino a aquel que juzga el videojuego. Aunque esto hace parecer que el problema radica en un tipo de ética subjetiva, objetivamente hablando es posible analizar las implicaciones éticas del contenido de un producto. Desde esta perspectiva, es posible plantear que el videojuego es bueno o malo por el tipo de obra que se ha creado y esta definición sería independiente de la valoración que cada quien le otorgará al producto (Górmez del Castillo, 2005).

Aristóteles (1999) cuestionaba los fines que se persiguen en cualquier acción humana y tal como él afirma en la Ética Nicomaquea: "siendo como son en gran número las acciones y las artes y ciencias, muchos serán de consiguiente los fines. Así, el fin de la medicina es la salud; el de la construcción naval, el navío; el de la estrategia, la victoria, y el de la ciencia económica, la riqueza" (p. 18). Desde esta perspectiva aristotélica, también resulta pertinente preguntar ¿cuál es el fin de la industria de los videojuegos? En términos aristotélicos podemos decir que el bien de cada actividad que realiza el hombre es aquello por cuya causa se pone en obra todo lo demás. Algunos de los fines de la industria de los videojuegos que se han declarado son la diversión, el aprendizaje, así como la estrategia de reclutamiento, en el caso del ejército norteamericano (Llorca, 2006).

Para algunos diseñadores de videojuegos el único fin al que debe orientarse un videojuego es que sea "divertido"; sin embargo, no solo la diversión es el fin del desarrollo de los videojuegos, también se encuentra "el aprendizaje del alumno" como 
fin de los videojuegos en el ámbito educativo (Etxeberria, 1998). Los fines del aprendizaje en los videojuegos están vinculados, por ejemplo, a una mejor comprensión histórica de los hechos de una revolución, descubrir el origen de los problemas de salud ambiental que recientemente han plagado a una comunidad, o aprender de forma interactiva cómo funcionan los campos electromagnéticos, creando un mundo en donde se le permite al alumno manipular cargas (Flores, 2009).

Según la opinión de Gee (2002), los videojuegos también promueven que los jugadores aprendan a resolver problemas, no se ve a las equivocaciones como errores, sino como oportunidades para mejorar. Los usuarios constantemente están generando hipótesis y poniéndolas a prueba. El videojuego alienta a los jóvenes a buscar mejores soluciones y enseña a enfrentar retos (Alfageme $\&$ Sanchez, 2002).

Además de desarrollar en el sujeto conocimientos y habilidades mediante los videojuegos, en investigaciones realizadas se ha reconocido que los videojuegos desarrollan una mejor coordinación entre el ojo y la mano, habilidades de visión espacial, favorecen que los jóvenes se sientan cómodos al utilizar la tecnología, promueven el pensamiento lógico y creativo, así como la comunicación y la cooperación (Dodig-Crnkovic \& Larsson, 2005). Estos diversos fines pueden ser considerados deseables para el ser humano ya que potencian las capacidades humanas (Etxeberria, 2008).

A pesar de lo antes expuesto, también se han reconocido potenciales efectos negativos de los videojuegos, como la disminución de la actividad física, la reducción de las conductas pro sociales, un bajo rendimiento escolar y el riesgo de adicción (Dodig-Crnkovic \& Larsson, 2005). Quienes critican los videojuegos violentos declaran que hay efectos indeseables en el ser humano, ya que a través del juego se acentúan las conductas antisociales y aumenta el nivel del miedo, al percibir el mundo como un lugar violento y rudo (Górmez del Castillo, 2005). Asimismo, el tiempo dedicado a los videojuegos tiende a ocupar el mayor espacio de ocio del sujeto cuando este no cuida mantener un equilibrio, llevándolo a ignorar otras formas de diversión en su tiempo libre (Aragón, 2011).

Partiendo del ideal aristotélico de que todo exceso es dañino, los jugadores que le dedican mucho tiempo al videojuego pueden llegar al punto de afectar su capacidad de socialización. De cierta forma, se abusa al vivir en el espacio virtual, de tal manera que puede desarrollar un vicio, pues se pierde la capacidad de ejercer la voluntad; el usuario es incapaz de dejar el videojuego. Ante esta situación, el uso del videojuego deja de enfocarse en el aprendizaje y limita el posible desarrollo del individuo (Sinde, Medrano \& Martínez, 2015).

\section{La regulación en la industria de los videojuegos}

Con relación a la regulación de la industria de los videojuegos, se ha encontrado que en los Estados Unidos esta actividad profesional está categorizada como una forma de expresión protegida por la primera enmienda de la Constitución de los Estados Unidos (Garfías, 2010). La enmienda prohíbe, entre otras cosas, cualquier ley que atente contra la libertad de expresión.

Para algunos jugadores el videojuego es una forma de diversión y para ellos es claro que en el mundo artificial se pueden hacer cosas que en el mundo real no son permitidas (Etxeberria, 2008). Se argumenta que para un gamer la violencia se queda en el videojuego y no va a las calles, por lo tanto, las posibilidades del contenido no deben ser restringidas ni reglamentadas (Górmez del Castillo, 2005). Sin embargo, si el videojuego para él representa en gran medida la realidad, esta distinción puede no ser tan clara e incluso se puede llegar a perder la línea entre ambas fronteras. Por otra parte, los diseñadores se justifican y buscan seguir las tendencias 
del mercado, las cuales se vinculan cada vez más con temas catalogados como maduros, es decir, que incluyen contenidos con violencia y sexualidad (Sinde, Medrano, \& Martínez, 2015).

Las empresas que producen este tipo de videojuegos argumentan su contenido considerando que este no es peor que el que se incluye en otras formas de entretenimiento, como son las películas o la música, señalando que los videojuegos solo reproducen la violencia que naturalmente se vive en la realidad (Takahashi, 2004). Empero, estos argumentos no han sido suficientes, por lo que la Entertainment Software Rating Board ha generado e instituido normas regulatorias sobre el contenido al que pueden ser expuestos los jugadores, clasificando los videojuegos de acuerdo con la edad de los posibles gamers (ESRB, 2017).

De esta forma, las empresas productoras de videojuegos deben incluir una descripción detallada del contenido de sus productos, considerando que cada juego solo puede contener aquello que resulta apropiado según el tipo de usuario al que se dirige. Pero, el problema de acceder a videojuegos no apropiados a la edad no se resuelve con una adecuada clasificación, ya que un gran número de videojuegos son usados por jugadores más jóvenes que la edad recomendada (Jenkins, 2010).

Aunque las empresas cumplan con la normatividad al marcar el contenido de los videojuegos, no se puede negar que en la realidad es muy difícil garantizar la aplicación de esta reglamentación. A pesar de que la edad promedio de los jugadores está alrededor de los 29 años, y que un 90\% de los videojuegos son vendidos a personas adultas, un gran número de estos van a dar a las manos de menores de edad (Takahashi, 2004). De acuerdo con un sondeo realizado por el National Institute of Media and the Family (NIMF, 2017), en los Estados Unidos menos del 5\% de los padres de familia entiende la restricción de uso del videojuego por su contenido. Por otra parte, en el mercado hay videojuegos con restricción de uso para adultos que son anunciados en medios en los que los menores de edad son consumidores (ESRB, 2017).

Corroborando lo anterior, si bien la industria puede incluir el contenido que quiera mientras este se encuentre señalado en la descripción del juego, esto no la desvincula de la responsabilidad social del producto que realiza. Sea cual sea la obra o el servicio que genera o presta una empresa, esta es responsable del fin último de lo que realiza, ya que, así como no se pueden negar los requerimientos de consumo de sus usuarios, tampoco se puede evitar la demanda legítima de responsabilidad que le exige la sociedad a la que presta sus servicios.

\section{El contenido como parte de la responsabilidad social de la industria de los videojuegos}

Según el Foro Económico Mundial (WEF, 2017), la Responsabilidad Social Empresarial (RSE) se refiere a todas aquellas prácticas que involucran una participación activa en iniciativas de bienestar social y desarrollo humano, que pretenden que las empresas puedan devolver a la sociedad un poco de lo mucho que esta les ha dado. De esta forma, la responsabilidad social ha dejado de ser una opción que las empresas pueden adoptar o no a su antojo, convirtiéndose en una legítima obligación que las haga cuestionarse sobre "cuánto ganan" y también sobre "cómo lo ganan", así como acerca del impacto que se genera al ganarlo (Crespo, 2010).

El concepto académico de la RSE no es realmente algo nuevo, pues desde 1953 en el libro de Howard Bowen, Social Responsibilities of the Businessman, se ha venido discutiendo sobre la manera idónea en que debe darse esta nueva visión de negocios en las empresas. Bowen (1953) consideraba que el actuar de las organizaciones tiene un impacto muy concreto en una gran parte de los ciudadanos, por lo que se necesita la vinculación de políticas y la toma de decisiones de las empresas, así como de los 
productos y servicios que generan, con base en los objetivos y valores de la sociedad.

Algo que cada vez se vuelve más claro para las organizaciones responsables es que realizar acciones aisladas no basta para desarrollar una verdadera cultura de RSE. Mientras las prácticas no impacten directamente en el modelo de negocios de la empresa o en los productos o servicios que oferta, las decisiones que se tomen se ven como una tendencia que, así como se aplican hoy en día, mañana simplemente puede dejar de seguirse (ETHOS-IARSE, 2014). De este modo, el contenido de los videojuegos no puede simplemente argumentarse porque la realidad sea violenta o la industria del entretenimiento oferte productos con contenidos semejantes, ya que esto no inhibe la responsabilidad de una empresa por ofrecer a sus usuarios productos de calidad, que respondan a sus necesidades y promuevan su desarrollo.

Según Kotler \& Zaltman (1971) una parte fundamental de la RSE es el diseño, la implementación y el control de programas pensados para influir en la aceptación de ideas sociales por medio de los productos, los servicios y la comunicación propia de la empresa, lo que resulta un punto determinante al momento de diseñar u ofertar bienes, debido a que estos tienen un inevitable impacto en sus consumidores.

Desde este enfoque, el contenido de los videojuegos, siendo el material ofertado, resulta ser un punto necesario de reflexión por parte de las compañías que desean no solo generar recursos económicos, sino también responder a la responsabilidad social que les corresponde. Para Acción Empresarial (2003), las organizaciones que ofrecen productos y servicios para las nuevas generaciones deben comprender que la sociedad en la que llevan a cabo sus actividades tiene un claro impacto en la manera en que se desempeñan, producen y son rentables, ya que hoy por hoy tener un buen modelo de negocios o un producto innovador no lo es todo. La industria debe poner atención a lo que pasa más allá de sus límites físicos, tomando decisiones y planteando acciones que incluyan las necesidades y las legítimas demandas de todos los grupos de interés, entre los que se encuentran sus usuarios, pero también las agendas sociales internacionales que promueven el desarrollo humano (Shen \& Benson, 2016).

Un modelo adecuado de RSE requiere transformar la cadena productiva en una cadena de valor compartido que impacte a todos los grupos relacionados con la empresa (WEF, 2017). De no ser así, las acciones propuestas pueden no ser coherentes, lo que es un valor natural e intrínseco de la responsabilidad social (Raufflet, Lozano, Barrera \& García, 2012). Querer ser amigable con el ambiente, promover la inclusión económica o defender los derechos humanos, pero ofrecer a su vez productos con un contenido cuestionable, hace que no se pueda considerar una empresa como verdaderamente responsable.

El fin de los videojuegos, como herramientas para la diversión, invita a que se reflexione sobre la manera en la que los jóvenes actualmente buscan pasar el tiempo de manera agradable (Llorca, 2006), ya que si el contenido del juego promueve la convivencia y el desarrollo de sensaciones placenteras, es natural y obvio que se consigue su objetivo, el cual puede cuestionarse si el fin último es despertar emociones violentas, ansiedad o angustia psicológica. La sociedad exige productos que cumplan sus fines últimos, pues de no ser así, ¿cuál es la razón de ser del bien o servicio prestado?

La industria de los videojuegos debe velar por la implementación y práctica de proyectos de responsabilidad en el contenido de sus productos, no solo como una forma de responder a esta demanda legítima de la sociedad, sino también como un elemento necesario para cumplir con la razón de ser de su arte u oficio. 


\section{Reflexión final}

Teniendo presente todos los puntos anteriormente señalados sobre la relevancia y el impacto del contenido de los videojuegos, y su relación con la responsabilidad de las empresas que los generan, se justifica la necesidad de una mayor orientación ética del trabajo de los productores de este tipo de bienes. El diseñador debe considerar el contenido, el propósito y las metas del videojuego y, a la par, lograr que su empresa tenga aceptación en la industria.

Los videojuegos deben articular el interés propio de divertir, con el interés social de promover el desarrollo humano. En otras palabras, el éxito de generar contenidos con responsabilidad social debe combinar el cometido de la empresa de lograr influir positivamente en el desarrollo de sus consumidores y grupos de interés. Un contenido responsable tiene claro su interés personal y de mercado, así como el fin último de conseguir diversión en el gamer, pero también la posibilidad de desarrollo y aprendizaje, responsabilizándose así de las implicaciones sociales de sus productos.

Es importante dejar claro que la responsabilidad social no implica que los intereses propios de la empresa sean ignorados a cambio del interés social, pues ambos intereses deben ser importantes. Los contenidos deben ver por ambas caras y objetivos, buscando que en un mismo producto se pueda ser exitoso y responsable al mismo tiempo.

En palabras finales, el objetivo del presente artículo era, más que dar respuestas sobre las implicaciones sociales de los videojuegos, abrir un espacio de reflexión sobre las posibilidades de contenidos socialmente responsables. De esta forma, a pesar de ser un texto de análisis bibliográfico, permite dar luz sobre este tema, considerando que sin importar el producto que se ofrezca o el servicio que se preste, siempre existe la oportunidad de actuar del modo correcto, convergiendo en el liderazgo de la industria y en la satisfacción de saberse socialmente responsables.

\section{Referencias}

Acción-Empresarial. (2003). El ABC de la responsabilidad social empresarial en Chile y el Mundo. Recuperado el 02 de mayo de 2016, de http://www. javeriana.edu.co/documents/245769/3057616/ El_ABC_de_la_RSE_en_Chile_y_el+mundo_

Alfageme, B. \& Sanchez, P. (2002). Aprendiendo habilidades con videojuegos. Comunicar, (19), 114-119.

Aragón, Y. (2011). Desarmando el poder antisocial de los videojuegos. Revista Electrónica Interuniversitaria de Formación de Profesorado, 14(2), 97-103.

Aristóteles. (1999). Ética Nicomaquea. México: Porrúa.

Bowen, H. (1953). Social Responsibilities of the Businessman. New York: Harper.

Cira, O. (2009). Ensayo sobre ética en los videojuegos. Manuscrito no publicado. Escuela de Negocios Ciencias Sociales y Humanidades. Tecnológico de Monterrey, Campus Monterrey. Monterrey, México.

Crespo, F. Entre el concepto y la práctica: responsabilidad social empresarial. Estudios Gerenciales, 26(117), 119-130.

Dodig-Crnkovic, G. \& Larsson, T. (2005). Game Ethics: Homo Ludens as a computer game designer and consumer. International Review of Information Ethics, 4, 20-23.

ESRB. (2017). Entertainment Software Rating Board. Recuperado en agosto de 2017 de Raitings guide: http://www.esrb.org/ratings/ratings_guide. aspx 
ETHOS-IARSE. (2014). Indicadores Ethos-IARSE para negocios sustentables y responsables. Buenos Aires: Programa Latinoamericano de Responsabilidad Social Empresarial (PLARSE).

Etxeberria, F. (2008). Videojuegos, consumo y educación. Teoría de la Educación. Educación y Cultura en la sociedad de la información, 9(3), 11-28.

Etxeberria, X. (1998). Videojuegos y educación. Comunicar, (10), 171-180.

Flores, F. (2009). Abriendo juego, abriendo mundos, Videojuegos Educativos. Recuperado el 28 de enero de 2010 de http://www.fernandoflores.cl/ node/2259

García, A. (2009). Ensayo sobre ética en los videojuegos. Manuscrito no publicado. Escuela de Negocios Ciencias Sociales y Humanidades. Tecnológico de Monterrey, Campus Monterrey. Monterrey, México.

Garfías, J. (2010). La industria del videojuego a través de las consolas. Revista Mexicana de Ciencias Politicas y Sociales, LII(209), 161-179.

Gee, J. (2003). What videogames have to teach us about learning and literacy. New York: Palgrave.

Górmez del Castillo, M. (2005). Violencia social y videojuegos. Pixel-Bit. Revista de Medios y Educación, (25), 45-51.

Jenkins, H. (2010). Reality Bytes: Eight Myths about video games debunked: The Video Game Revolution. Recuperado el 28 de enero de 2010 de http://www. pbs.org/kcts/videogamerevolution/impact/myths. html

Kotler, P. \& Zaltman, G. (1971). Social Marketing: An approach to planned social change. Journal of marketing, 3-12.

Llorca, Á. (2006). Los videojuegos, marcadores de tendencias en el ocio tecnológico. Comunicar, (27), 79-84.
McCormick, M. (2001). Is it wrong to play violent video games? Ethics and Information Technology, 3, 277-287.

NIMF. (2017). National Institute of Media and the Family. Recuperado en agosto de 2017 de About: www.mediafamily.org

Peláez, B. (2009). Futbol y videojuegos: reinventando el juego. Razón y Palabra, 14(69), 1-14.

Platón. (2007). Diálogos. México: Editorial Porrúa Raufflet, E., Lozano, J., Barrera, E. \& García, C. (2012). Responsabilidad Social Empresarial. México: Pearson.

Rodríguez, V. \& Pestano, J. (2012). Los videojuegos en España: una industria cultural incipiente. Ámbitos, (21), 361-379.

Shen, J. \& Benson, J. (2016). When CSR is a social norm. How socially responsible human resource management affects employee work behavior. Journal of Management, 42(6), 1723-1746.

Simone, B. \& López, C. (2008). Breve historia de los videojuegos. Athenea Digital. Revista de pensamiento e investigación social, (14), 159-179.

Sinde, J., Medrano, C. \& Martínez, J. (2015). Transmisión de valores en adolescentes: un análisis con videojuegos. Revista Latina de Comunicación Social, (70), 230-251.

Solís, J. (2008). Responsabilidad social empresarial: un enfoque alternativo. Análisis Económico, XXIII(53), 227-252.

Takahashi, D. (2004). Ethics of Game Design. Gamasura. Recuperado el $1^{0}$ Dic. de 2007 de $<$ http://games.cs.ualberta.ca/299/Gamasutra.pdf>

WEF. (2017). World Economic Forum. Recuperado https://www.weforum.org/ 


\section{Datos de filiación}

Florina Guadalupe Arredondo Trapero: doctora en Economía y Dirección Empresarial, Universidad de Deusto, España. Se desempeña como profesora de la Escuela de Humanidades y Educación del Tecnológico de Monterrey, en Monterrey, México. Sus líneas de investigación son los temas de responsabilidad social empresarial.

Correo electrónico: farredon@itesm.mx
José Carlos Vázquez Parra: doctor en Estudios Humanísticos por el Tecnológico de Monterrey, en Monterrey. Se desempeña como profesor de la Escuela de Humanidades y Educación del Tecnológico de Monterrey, en Guadalajara; su línea de investigación más importante es la Ética empresarial y la racionalidad del comportamiento. Correo electrónico: jcvazquezp@itesm.mx 